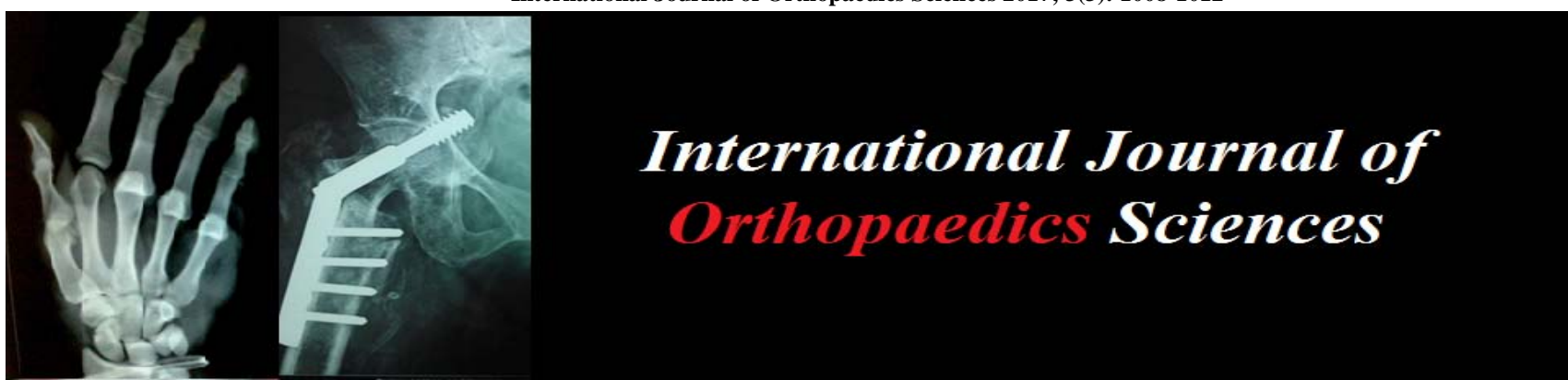

ISSN: $2395-1958$

IJOS 2017; 3(3): 1008-1012

(C) 2017 IJOS

www.orthopaper.com

Received: 19-05-2017

Accepted: 20-06-2017

Dr. Manish Kumar

Assistant Professor,

Department of Orthopaedics, IGIMS, Patna, Bihar, India

Dr. Rakesh Kumar

Senior Resident,

Department of Orthopaedics,

IGIMS, Patna, Bihar, India

Dr. Rajesh Kumar Ranjan Senior Resident,

Department of Orthopaedics, IGIMS, Patna, Bihar, India

Dr. Ashutosh Kumar Senior Resident,

Department of Orthopaedics, IGIMS, Patna, Bihar, India

Dr. Farman Ali Senior Resident, Department of Orthopaedics, IGIMS, Patna, Bihar, India

Dr. Santosh Kumar Professor, Department of Orthopaedics, IGIMS, Patna, Bihar, India

Correspondence

Dr. Manish Kumar

Assistant professor,

Department of Orthopaedics,

IGIMS, Patna, Bihar, India

\section{Results of pronator quadratus pedicle bone graft as primary procedure for treatment of scaphoid nonunion}

\author{
Dr. Manish Kumar, Dr. Rakesh Kumar, Dr. Rajesh Kumar Ranjan, \\ Dr. Ashutosh Kumar, Dr. Farman Ali and Dr. Santosh Kumar
}

DOI: https://doi.org/10.22271/ortho.2017.v3.i3n.149

Abstract

Pedicle bone graft as primary procedure for treatment of scaphoid nonunion. Pedicle bone graft use in delayed and nonunion of fractures and avascular necrosis scaphoid fracture. The most common indications for Pedicle bone graft have been for scaphoid nonunion, lunatomalacia (Kienböck's disease), and osteonecrosis of the scaphoid (Preiser's disease). Advantages over non vascularized bone graft have been established. Pedicle bone graft provide improved blood flow, osteocyte preservation, and accelerated healing rates. Local pedicle VBGs are the most commonly used methods. They are technically less demanding than are free VBGs and are associated with less morbidity. In managing osteonecrosis, most surgeons would agree that VBG should be reserved for scaphoid nonunion with an intact cartilaginous shell and no collapse. In treating scaphoid pathology, indications for pedicle bone graft include fractures/nonunions with proximal pole avascular necrosis and/or small proximal pole fragments.

Keywords: Pedicle bone graft, osteonecrosis, nonunion

\section{Introduction}

Among all the carpal bones, scaphoid is the most commonly fractured bone ${ }^{1}$. It is seen in young and physically active adults. 5 to $30 \%$ of scaphoid fractures involve towards non-union $[2,3]$. Most common reason for this is the less blood supply to the proximal pole of the scaphoid. Proximal $80 \%$ of scaphoid is supplied by dorsal scaphoid branch while distal $20 \%$ is supplied by volar scaphoid branches of the radial artery or superficial palmer arch. Other reason for nonunion is its peculiar anatomy, is that it lacks periosteal covering. More than $80 \%$ of the scaphoid surface is covered by articular cartilage while the remaining surface acts as sites for vascular supply. Due to absence of periosteum, union in scaphoid fracture occurs by primary bone healing resulting in minimal callus formation and biomechanically weak early union ${ }^{[4]}$.

In primary care set up, scaphoid fractures are frequently missed because of minimal initial symptoms. This leads to late diagnosis and subsequently delayed immobilization. This delayed treatment results in a nonunion upto $40 \%{ }^{5}$ to $88.1 \%$. Fracture pattern is the next cause responsible for nonunion of scaphoid fractures. Undisplaced fractures proceed to nonunion in $5 \%$ to $10 \%$ of cases. While displacement of fracture fragments $(\geq 1 \mathrm{~mm})$ or angulation $>15^{0}$ are accepted risk factors for nonunion. Similarly, oblique fractures a high rate of nonunion or malunion if treated without accurate reduction and fixation.

Scaphoid bone acts as a bridge connecting both the proximal and distal rows ${ }^{7}$. Through its proximal and distal ligamentous attachments, the scaphoid serves to coordinate and smoothen the motions of the proximal and distal rows. After its fracture, dissociation of proximal and distal carpal row occurs which permits the natural tendency of the two carpal rows to fail by collapsing. Chronic scaphoid nonunion progresses to carpal collapse also known as "Scaphoid Nonunion Advanced Collapse" and degenerative arthritis ${ }^{[2,3,8]}$. Initially, arthritis is limit to the articulation between radial styloid and distal pole of scaphoid. Later on, it affects the radial scaphoid fossa as well as the midcarpal joint. 
The ideal treatment of scaphoid nonunion is still controversial. Several techniques with varying success rates have been used for the treatment of scaphoid nonunions. The most widely accepted approach is a combination of bone grafting and internal fixation. The rate of osseous union depends on the stability and vascularity of the proximal fragment ${ }^{[9]}$. Conventional bone grafts yield good results in viable proximal pole fractures. Vascularized bone grafts are used secondarily in cases where conventional bone grafts fail most often due to AVN of proximal pole.

The purpose of the study was to evaluate the effectiveness of pronator quadratus pedicled bone graft as the primary treatment method for scaphoid nonunion. We present the surgical results of seventeen patients with scaphoid nonunion treated with pronator quadrates pedicled bone graft and Herbert screw fixation.

\section{Material and methods}

Between January 2011 to July 2017, 17 patients meeting the inclusion criteria were treated for scaphoid nonunion by pronator quadratus muscle pedicle based vascularized bone graft and Herbert Screw fixation. Demographic data regarding age, sex, anatomical location of fracture, hand dominance and duration between injury and surgery, type of nonunion and form of primary treatment received by the patient were noted. Patients underwent detailed clinical evaluation for localization of tenderness (dorsally in anatomical snuffbox and volarly over scaphoid tubercle), range of motion of affected wrist in comparison to normal wrist and hand grip strength. Preoperative radiological examination included the standard postero anterior, lateral and oblique view of the affected wrist and an anteroposterior clenched fist view to stress the scapholunate ligament.

\section{Inclusion/Exclusion Criteria}

Patients were included if they met the following criteria: (1) persistent tenderness, (2) incomplete trabecular bridging on three X-ray views, (3) injury that had occurred at a minimum of 6 months prior to surgery and not more than 5 years. Patients were excluded if they exhibited avascular necrosis of the proximal pole, humpback deformity, mid-carpal instability or carpal collapse. Patients were also excluded if they had undergone previous surgery for scaphoid fixation with conventional bone graft.

\section{Surgical technique}

A linear incision was made centered over the distal radius and the scaphoid tuberosity. The

radioscaphocapitate ligament was incised. The nonunion site was exposed and the fibrous material was curetted until normal bone was visible. In viable proximal fragments bleeding points were seen even with the tourniquet inflated. Then, pronator quadratus muscle's distal insertion over the radius was identified and bone block of $5 \times 10 \mathrm{~mm}$ was outlined close to abductor pollicis longus tendon. Graft was separated with a fine osteotome taking utmost care not to detach the muscle from it. The muscle was then dissected towards the ulna to secure a $10 \mathrm{~mm}$ thick pedicle. In cases, where the muscle was too tight to allow easy transfer of the pedicled bone, the ulnar origin of the muscle was dissected subperiostally from the distal ulna through an additional incision.

A traction force was applied over the thumb to align the proximal and distal fragments fixed with a Herbert screw. The anterior surface of the scaphoid was made raw both in the proximal and distal fragments. The pedicled bone block was fixed to the raw surface using 3-0 poly glycolic acid suture. The vascularity of the graft was verified by inspection of bleeding from it after deflating the tourniquet. The radio scapho capitate ligament was sutured back. The skin incision was closed without tension and a long arm cast with the thumb in opposition was applied for 6 weeks, followed by a short cast for next 6 weeks. Physiotherapy and active exercises were started after 3 months.

\section{Postoperative course}

Patients were followed up every six weeks and serial radiographs were taken which included postero anterior, lateral and oblique views. Union was defined as bony trabeculae crossing the nonunion site and absence of sclerosis at the union site. Clinical examination included the assessment of pain with palpation (clinical union), and during rest and activities. ROM was measured using a goniometer. Grip strength was assessed using a dynamometer and compared with the uninjured contralateral extremity. Clinical outcome was assessed using the modified Mayo Wrist Score and Disabilities of Arm, Shoulder and Hand score.

\section{Results}

All patients were followed up for at least 1 year. The mean follow-up period in our study was 25.5 months (12-54 months). The mean age of patients in our study was $31.7 \pm 7.9$ years. Fourteen patients were male $(82 \%)$ and three was female. There were five fractures of the proximal third of the scaphoid and 12 waist fractures. The mean time from injury to surgery was 8.2 months (range, 6 to 12 months). At the moment of the surgery, 11 cases had fibrous nonunions and 6 cases had sclerotic pseudarthrosis. All the patients achieved bony consolidation after an average of 9.8 weeks (range 6 to 12 weeks). The radiologic criterion of bony consolidation included: disappearance of fracture line, bridging bone trabeculae and restoration of normal bone density. The cast immobilisation was removed when radiologic union was achieved. 15 patients had no pain after the immobilization was removed and 2 patients had mild pain, which subsided after physiotherapy.

The mean flexion was $81^{\circ}$ (range, $70^{\circ}-90^{\circ}$; preoperative mean, $57^{\circ}$ ). The mean extension was $74^{\circ}$ (range, $65^{\circ}-80^{\circ}$; preoperative mean, $59^{\circ}$ ). Ulnar deviation averaged $39^{\circ}$ (range, $30^{\circ}-45^{\circ}$; preoperative mean, $32^{\circ}$ ) and radial deviation averaged $18^{\circ}$ (range, $15^{\circ}-20^{\circ}$; preoperative mean, $12^{\circ}$ ). Combined pronation and supination were $177^{\circ}$ (range, $170^{\circ}-$ $185^{\circ}$; preoperative mean, $171^{\circ}$ ). The mean grip strength was $99 \%$ (range, 95\% 100\%) compared with that of the contralateral healthy extremity, which had improved from a preoperative mean of $67 \%$.

There were no early complications of surgical site infection or sensory disturbances in the area of the radial nerve. There were also none late septic complications. No radiographic progression of arthritis was noted in any patient within the available follow-up time of 25.5 months. No arthritic changes were noted at the dorsal ridge of the radius (where the graft had been harvested). Mean postoperative DASH score was 10.2 (range, 0 to 52). All the 17 patients returned to their previous level of activity. All the patients were satisfied with the late clinical and functional results.

\section{Statistical analysis}

Statistical analysis was performed using the Student $t$ test to compare preoperative and postoperative values; $p$ values less than .05 were considered statistically significant. 
Table 1: Demographic details of patients

\begin{tabular}{|c|c|c|c|c|c|c|}
\hline Case No. & Age (yrs) & Gender & Side & Duration between injury and surgery & Fracture Site & Type of Nonunion \\
\hline 1 & 24 & $\mathrm{M}$ & $\mathrm{R}$ & 6 & proximal pole & fibrous \\
\hline 2 & 33 & $\mathrm{M}$ & $\mathrm{R}$ & 10 & waist & fibrous \\
\hline 3 & 47 & $\mathrm{M}$ & $\mathrm{L}$ & 9 & waist & sclerotic \\
\hline 4 & 25 & $\mathrm{M}$ & $\mathrm{R}$ & 7 & waist & fibrous \\
\hline 5 & 37 & $\mathrm{~F}$ & $\mathrm{R}$ & 7 & proximal pole & fibrous \\
\hline 6 & 22 & $\mathrm{M}$ & $\mathrm{R}$ & 6 & waist & sclerotic \\
\hline 7 & 43 & $\mathrm{M}$ & $\mathrm{L}$ & 8 & waist & sclerotic \\
\hline 8 & 27 & $\mathrm{M}$ & $\mathrm{L}$ & 11 & waist & fibrous \\
\hline 9 & 26 & $\mathrm{M}$ & $\mathrm{R}$ & 8 & proximal pole & fibrous \\
\hline 10 & 29 & $\mathrm{M}$ & $\mathrm{R}$ & 10 & waist & fibrous \\
\hline 11 & 33 & $\mathrm{M}$ & $\mathrm{R}$ & 12 & waist & fibrous \\
\hline 12 & 31 & $\mathrm{~F}$ & $\mathrm{R}$ & 8 & waist & fibrous \\
\hline 13 & 43 & $\mathrm{M}$ & $\mathrm{L}$ & 9 & waist & sclerotic \\
\hline 14 & 28 & $\mathrm{M}$ & $\mathrm{R}$ & 8 & proximal pole & sclerotic \\
\hline 15 & 38 & $\mathrm{~F}$ & $\mathrm{R}$ & 7 & waist & fibrous \\
\hline 16 & 19 & $\mathrm{M}$ & $\mathrm{L}$ & 7 & waist & fibrous \\
\hline 17 & 34 & $\mathrm{M}$ & $\mathrm{R}$ & 6 & & \\
\hline
\end{tabular}

Table 2: Preoperative and Postoperative parameters

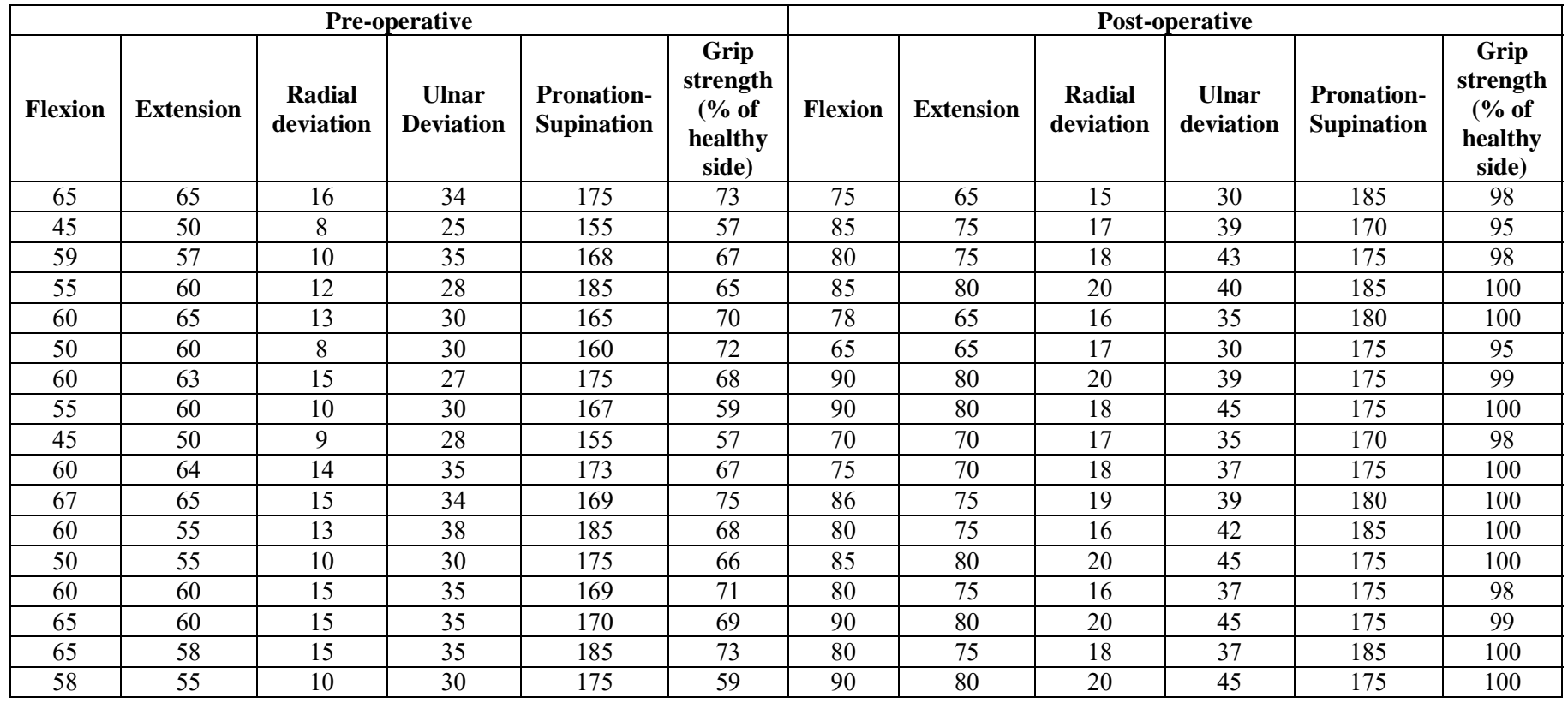

\section{Figures}

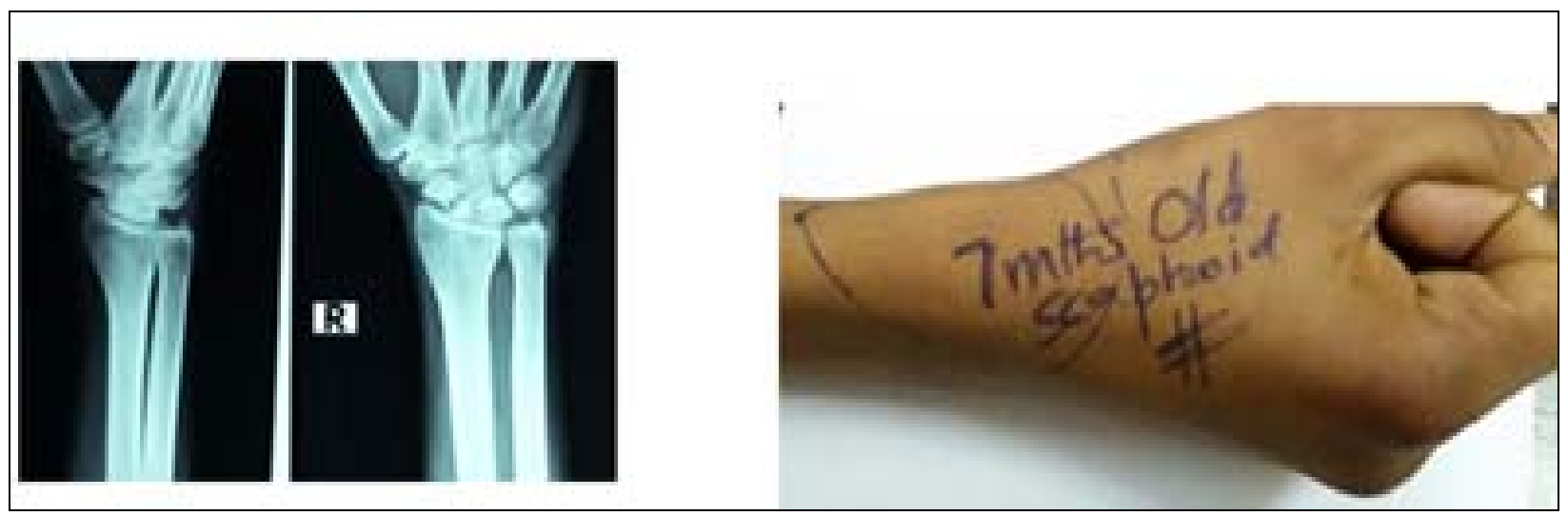

Fig 1: Pre op x ray and clinical presentation of 7 month old non union scaphoid fracture 

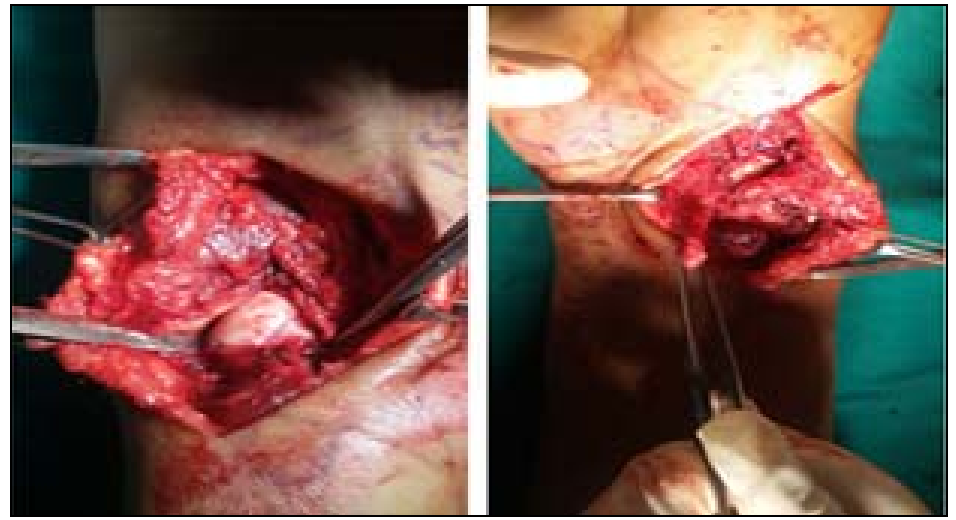

Fig 2: Intra op showing use of pronator quadratus pedicled bone graft for treatment of scaphoid nonunion

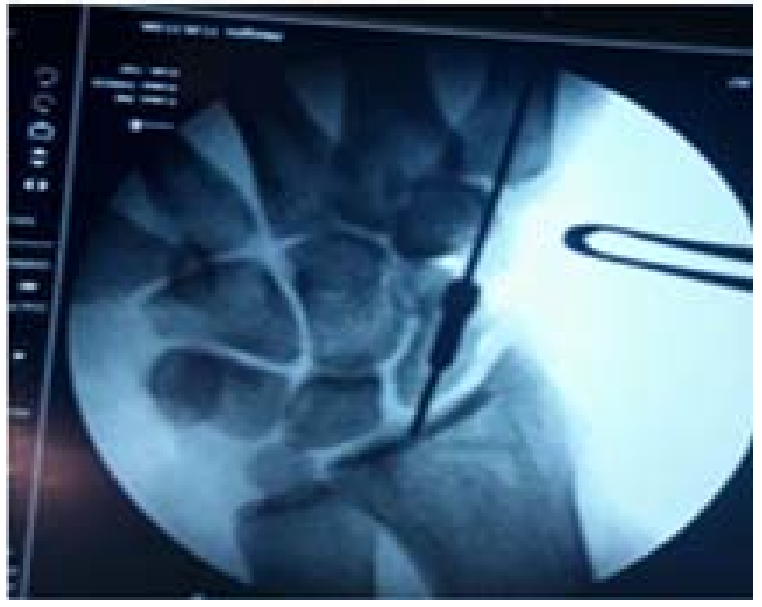

Fig 3: Intra op image showing herbert screw fixation

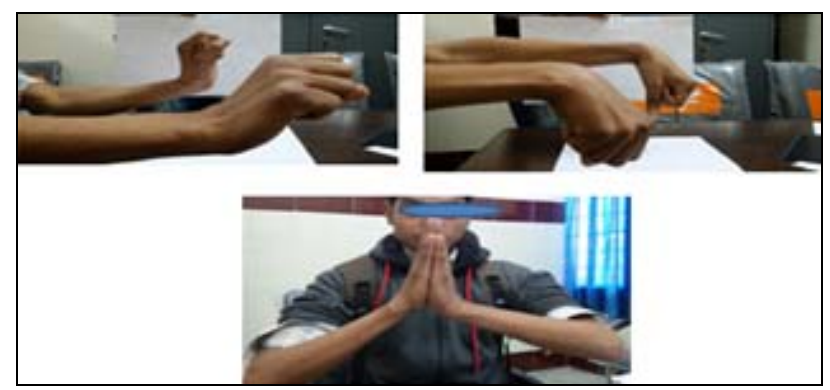

Fig 4: Three months post op range of motion of wrist

\section{Discussion}

Management of scaphoid nonunion is a challenging task for an orthopaedic surgeon. Many surgical procedures have been employed, which reflects the lack of satisfaction or consistency with any one strategy. In cases of established nonunion, bone grafting is advocated to facilitate healing and correction of any carpal malalignment. Most important intraoperative step in the management of scaphoid nonunion is excision of the psudoarthrosis and fibrous scar tissue at the nonunion site. In doing so, a bony defect is inevitably created. This defect can be filled with either free bone graft or vascularized one. When a nonvascularized bone graft is put at the nonunion site, the risks of its absorption and failure of the graft to revascularize are high. While a bone graft with an intact pedicle of blood supply remains viable, in addition, it unites directly with the recipient bone without necessity of revascularization or replacement by creeping substitution ${ }^{[10,}$ ${ }^{11]}$. It is also a ready source of vascular osteogenic tissue which sprouts new outgrowths to revascularize the avascular recipient bone tissue.

Vascularized bone grafts are usually advocated for failure of conventional techniques. But in cases of nonunion with AVN of proximal pole, most of the surgeons advocate use of primarily vascularized graft. Vascularized bone grafts can be derived from several different locations. The most commonly used bone grafts are from the distal radius.

The earliest report of a vascularized pedicle bone graft applied to carpal pathology was by Roy-Camille in $1965^{[14]}$. Using the scaphoid tubercle on an abductor pollicis brevis muscle pedicle, he performed a vascularized bone graft to assist successfully in the healing of a scaphoid waist delayed union. In 1979, Hori et al. initially described superficial radial artery based pedicle graft in a canine model. Active proliferation of blood vessels and new-bone formation was found when the vascular pedicle was implanted into the bone ${ }^{[15]}$. In 1983, Braun described a volar distal radius bone graft based on a pronator quadratus muscle/anterior interosseous artery pedicle and successfully treated five scaphoid nonunions ${ }^{[16,17]}$. In 1984, Chacha reported the use of bone graft with a vascular pedicle from the pronator quadratus on two patients with nonunion of scaphoid with avascular necrosis ${ }^{[18]}$. In 1988, Kawai and Yamamoto reported on a volar pronator quadratus pedicle with which they achieved union in all eight patients with a scaphoid non-union ${ }^{[19]}$.

In 2015, Lee SK reported on 27 patients with scaphoid nonunion who were treated with a pronator quadratus pedicled vascularized bone graft and a headless compression screw. They achieved bony union in all the 27 patients $(100 \%)$ in a mean of 11.5 weeks ${ }^{[20]}$. There was improvement in mean radiolunate and scapholunate angles. Average range of motion and modified Mayo score and DASH score had improved. In 2015, Bogdan Obada reported on six ununited scaphoid fractures and treated with pronator quadratus pedicled bone graft and Herbert-screw or Kirschner-wires fixation ${ }^{[21]}$. They achieved radiographic consolidation in all the six cases after an average of 9.8 weeks. The final results according to Mayo Clinic Wrist Score were excellent in 3 cases and good in 3 cases.

\section{Conclusion}

The technique used in our series is an easy method and provides excellent results while needing no special equipment. We recommend it as primary treatment procedure for scaphoid non-union irrespective of status of vascularity of proximal pole due to the quality of the functional results and the speed of consolidation. This study had several limitations. It was a retrospective case study with no control group. The 
numbers of cases were relatively small due to the fact that scaphoid nonunion is relatively rare, allied to the difficulty in getting patients to return for evaluation. CT scan was not performed for assessing union of scaphoid as CT provides better accuracy than the plain radiographs.

\section{Conflict of Interest}

None

\section{Reference}

1. Amadio P, Moran SL. Fractures of the carpal bones. In: Green DP, Hotchkiss RN, Pederson WC, editors. Green's operative hand surgery. 5th ed. Philadelphia, PA: Elsevier Churchill Livingstone; 2005, 711.

2. Kawamura K, KC. Chung, Treatment of scaphoid fractures and nonunions J Hand Surg Am, 2008; 33(6):988-997

3. AN Larson, AT Bishop, AY Shin. Dorsal distal radius vascularized pedicled bone grafts for scaphoid nonunions. Tech Hand Up Extrem Surg, 2006; 10(4):212-223

4. Slade JF 3rd, Dodds SD. Minimally invasive management of scaphoid nonunions. Clin Orthop Relat Res. 2006; 445:108-19.

5. Langhoff $\mathrm{O}$, Andersen JL. Consequences of late immobilization of scaphoid fractures. J Hand Surg $\mathrm{Br}$ 1988; 13(1):77-79.

6. Eddeland A, Eiken O, Hellgren E, Ohlsson NM. Fractures of the scaphoid. Scand J Plast Reconstr Surg. 1975; 9(3):234-239.

7. Kuschner SH, Lane CS, Brien WW. Scaphoid fractures and scaphoid nonunion. Diagnosis and treatment. Orthop Rev. 1994; 23:861-71.

8. Szabo RM, Manske D. Displaced fractures of the scaphoid. Clin Orthop 1988; 230:30-8.

9. Gabl M, Reinhart C, Lutz M, Bodner G, Rudisch A, Hussl $\mathrm{H}$, Pechlaner S. Vascularized bone graft from the iliac crest for the treatment of nonunion of the proximal part of the scaphoid with an avascular fragment, J. Bone Joint Surg., 1999; 81-A(10):1414-1428

10. Mack GR, Bosse MJ, Gelberman RH, Yu E. The natural history of scaphoid nonunion. J Bone Joint Surg Am 1984; 66:504-9.

11. Ruby LK, Leslie BM. Wrist arthritis associated with scaphoid nonunion. Hand Clin 1987; 3:529-37

12. Russe O. Fracture of the carpal navicular. Diagnosis, nonoperative treatment, and operative treatment. J Bone Joint Surg Am 1960; 42:759-68

13. Fernandez DL. A technique for anterior wedge-shaped grafts for scaphoid nonunions with carpal instability. J Hand Surg Am 1984; 9(5):733-7.

14. Roy-Camille R. Fractures and pseudoarthrosis of the scaphoid: utilization of pedicled graft. Actual Chir Orthop R Poincare 1965; 4:197-214.

15. Hori Y, Tamai S, Okuda H, Sakamoto H, Takita T, Masuhara K. Blood vessel transplantation to bone. J Hand Surg Am 1979; 4(1):23-33. 